\title{
Integrating organic light-emitting diode and field-effect-transistor in a single
}

\section{device}

Bin Wei, Jun Wang*

Key Laboratory of Advanced Display and System Applications, Ministry of Education, Shanghai University, P. O.

B. 143, 149 Yanchang Road, Shanghai University, Shanghai, 200072, P. R. China

Chong Li, Aoi Shimada, Musubu Ichikawa, Yoshio Taniguchi

Department of Functional Polymer Science, Shinshu University, 3-15-1 Tokida, Ueda, Nagano 386-8567, Japan

Taketomi Kamikawa

Institute of Frontier Device, Seiko Epson Corporation, 281 Fujimi, Fujimi-machi, Suwa-gun, Nagano, 399-0293,

Japan

\section{Abstract}

We have investigated organic light-emitting diodes (OLEDs) with a structure of field-effect-transistor (FET) using $\mathrm{MgAg}$ as source and drain electrode, respectively. These devices were found to present both a typical switchable behavior of FET and an electro-optical transfer characteristic of OLEDs. Five organic layers were employed in devices that were expected to play each role for OFET and OLED, respectively. In a result, the FET unipolar behavior has been demonstrated experimentally. Furthermore, FET characteristics were discussed based on an equivalent circuit.

\footnotetext{
*Email address: wangj@shu.edu.cn
} 


\section{Introduction}

Organic semiconductor devices have received much attention due to intensive applications of organic light-emitting diodes (OLEDs) in lightening, flat panel displays, and optically pumped organic thin films in stimulated emission [1-3]. Besides, organic field effect transistors (FETs) based on organic thin film or single crystals (such as tetracene, pentacene or rubrene) are also one of the topics that have attracted great attention in these years [4-7]. Furthermore, some research groups have reported field-effect transistor (FET) driven OLEDs and organic light-emitting transistors (OLETs) [8-12]. Since transistors can control the amount and the species (hole or electron) of injection carriers by the third electrode and employing OLETs will make drive circuits of displays simpler than using organic light-emitting diodes, OLETs are expected to become promising in the fields of high performance light-emitting devices. However, since the emission in OLETs mainly radiate toward the edge of film or crystal under wave-guided, the light intensity is relatively weak and emission spectrum is difficult to observe [13]. Furthermore, the integration of two kinds of devices (OFET and OLED) on a substrate has been reported [14].

In this paper, we reported an OLED with a FET structure by which will overcome the disadvantages of OLETs, such as edge emission and light quenching near metal drain electrode. Compared to TFT driven OLEDs, our devices present both a unipolar FET behavior and an electro-optical transfer characteristics of OLEDs. The FET behavior has been observed from an OLED with a strong surface emission mode.

\section{Experimental}

We have developed a type of OLED with a FET structure, as shown in Fig. 1. For top - contact configuration we defined, as shown in Fig. 1, a indium-tin-oxide (ITO) glass substrate with $150 \mathrm{~nm}$ was served as a gate electrode, and a $150 \mathrm{~nm}$ of $\mathrm{MgAg}$ as both drain and source electrode. The structure of organic layers in devices is 2,5-bis(6'-(2',2"-bipyridyl))-1,1-dimethyl-3,4-diphenylsilole (PyPySPyPy, $80 \mathrm{~nm})$ / bathocuproine (BCP, 10nm) / tris(8-hydroxyquinoline)aluminum (Alq, 30nm) 
/4,4'-bis[N-(1-napthyl)-N-phenyl-amino]-biphenyl (NPB, 60nm) / tris [2-naphthyl (phenyl) amino] triphenylamine (2-TNATA, 220nm). We selected 2-TNATA to function as a hole injection and transport layer, and PyPySPyy as electron transport layer, due to their relatively high carrier mobility [15-16], for which the devices with $400 \mathrm{~nm}$ of organic layers can be operated under high voltages without degradation. In addition, the BCP was employed as a hole block layer to prevent hole leak into electron transport layer. These organic layers and MgAg electrode (9:1 mass ratio) were processed by thermal deposition. The deposition rates were typically $0.1 \mathrm{~nm} / \mathrm{s}$ and $0.5 \mathrm{~nm} / \mathrm{s}$ for organic materials and metal cathode, respectively.

A shadow mask with one thin tungsten line of diameter $30 \mu \mathrm{m}$ was carefully mounted on the organic layers to form a top-contact configuration. On the other hand, we have fabricated a bottom-contact configuration with a very thin channel on ITO. We have etched line ITO to achieve a drain and a source electrode by the following procedures. First, we used electron beam (EB) lithography (Tokyo technology Co. Ltd) to form a narrow pattern with an order of $\mu m$ on a cleaned nonluminescent glass substrate (beam current is $250 \mathrm{pA}$, exposure time is $0.75 \mu \mathrm{S}$, lithography field is the order of $\mu \mathrm{m}^{2}$, resolution is $0.02 \mu \mathrm{m} / \mathrm{pixel}$ ). In addition, EB resist (ZEP520A) was used to carry out processing procedure. Next, a thick LiF layer with $300 \mathrm{~nm}$ was thermally deposited on the glass substrate with EB pattern. Then the substrate was immersed in N, N-dimethylacetoamide solution and ultrasonic wave treatment was carried out to remove $\mathrm{LiF}$ onto EB resist. Subsequently, opaque metal $\mathrm{Cr}$ was deposited onto the substrate and then LiF was liftoffed by a treatment in hot water with ultrasonic wave for 30 minutes. Finally, using the completed Cr mask with narrow channel onto an ITO substrate, which has been spincoated with photoresist agent under UV exposure, we etched the substrate in mixed acid for 35 minutes and obtained an ITO pattern with a very narrow channel.

The electric characteristics of devices were measured with two source electrometers (Advantest, $\mathrm{R} 6245)$ in vacuum $\left(10^{-3} \mathrm{~Pa}\right.$ or less) environment at room temperature. Two $\mathrm{Au}$ wires as probes were placed onto gate and drain contact carefully by observing optical microscope (Keyence digital HF 
microscope $\mathrm{VH}-8000$ ). We investigated the device characteristics by scanning $\mathrm{V}_{\mathrm{D}}$ from 0 to $20 \mathrm{~V}$ at a constant of $\mathrm{V}_{\mathrm{G}}(15 \mathrm{~V})$ with a step of $0.5 \mathrm{~V}$ (ITO as drain contact for bottom configuration and gate contact for top configuration).

\section{Results and discussion}

These devices exhibited typical OLED characteristics when applying voltage on source and gate contact. The electric and luminescence characteristics of OLED were showed in Fig. 2. An OLED (size: $4 \mathrm{~mm}^{2}$ ) with ITO as an anode and MgAg (Source contact in OFET) as a cathode has been presented. Results found that the turn-on voltage to observe the luminescence of $0.1 \mathrm{~cd} / \mathrm{m}^{2}$ was $7.5 \mathrm{~V}$, and maximum luminance of $5252.1 \mathrm{~cd} / \mathrm{m}^{2}$ was realized at $29.5 \mathrm{~V}$. The current density and luminance were $0.36 \mathrm{~mA} / \mathrm{cm}^{2}$ and $6.3 \mathrm{~cd} / \mathrm{m}^{2}$ at $10 \mathrm{~V}, 2.42 \mathrm{~mA} / \mathrm{cm}^{2}$ and $46.0 \mathrm{~cd} / \mathrm{m}^{2}$ at $15 \mathrm{~V}$.

It is noted that typical transistor characteristics can be clearly observed, too. When applying positive drain and gate voltage, electrons are injected into organic layers from $\mathrm{MgAg}$ contact. Figure 3 shows the family curves $\left(I_{D}-V_{D}\right)$ of our devices. The linear and saturation regiem may be observed with the increase in $V_{D}$. Electrons are injected from the source contact, and extracted from drain contact. The depletion region under drain contact is gradually enlarged with the increase in $V_{D}$, leading to the form of saturation current. From the transfer curves $\left(\mathrm{I}_{\mathrm{D}}-\mathrm{V}_{\mathrm{G}}\right)$, the switch characteristics of transistors are displayed with the increase of $\mathrm{V}_{\mathrm{G}}$. Here, electrons are accumulated at the interface of Alq and NPB/2-TNATA by applying positive gate voltage. Therefore, current device with OLED structure also showed typical characteristics of field-effect transistors although convention gate insulator and active layer have not been defined in current devices. The function of each layer in devices was also showed in Fig. 1. According to the configuration and operation mechanism of FET, the five organic layers are believed to act as different function. The upper two layers (BCP and PyPySPyPy) will serve as two charge (electrons) injection layers, the light emitting layer (Alq) as the active layer in transistors, and the last two layers (NPB/2-TNATA) as the gate dielectric layer. Thus, these devices also exhibit transistor behavior. Furthermore, noted that electron accumulation mode was operated by using $\mathrm{MgAg}$ 
as source/drain contact for easily electrons injection due to its low work function. On the other hand, a similar device with ITO as source and drain contact and MgAg as gate electrode in inversion structure displays hole accumulation mode due to easily hole injection. Despite of the output current is low due to low mobility of $\mathrm{Alq}_{3}$ when compared with conventional organic transistors [17], the typical device characteristics will present a new approach to developing organic light-emitting transistors. Further optimizations about devices performance are in progress.

We have established an equivalent circuit to discuss the FET behavior. Similar to one OLED [17], the equivalent circuit of the device with top-contact configuration used in this study can be schematically described in Fig. 4(a). The internal resistance of organic layer for source-drain contact, source-gate contact and drain-gate contact in device was assumed to be $r, R_{1}, R_{2}$, respectively. We described the currents of three contacts, $I_{D}, I_{G}$ and source current $\left(I_{S}\right)$ using the following expression:

$$
I_{G}=\frac{V_{G}}{R_{1}}+\frac{V_{G}-V_{d}}{R_{2}} \quad I_{D}=-\frac{V_{G}-V_{D}}{R_{2}}+\frac{V_{D}}{r} \quad I_{s}=-\frac{V_{G}}{R_{1}}-\frac{V_{d}}{r}
$$

In Eq. (1), the $I_{S}$ is considered to be equal to the negative of the sum of $I_{D}$ and $I_{G}$, which have been recorded experimentally. We plotted the relative change of $I_{S}$ at some $V_{D}=V$ with respect to the value of $I_{S}$ at $V_{D}=0 \mathrm{~V}$ against the gate voltages, as shown in Fig. 4(b). It was clearly observed the $V_{D}$ dependence of $I_{S}$ at a low drain voltage region, as described by Eq. (1), although the value of $I_{S}$ is at the order of $\mu \mathrm{A}$. This demonstrates that a FET behavior has been obtained in addition to OLED characteristics, particularly at the low drain voltage condition. Moreover, it is noted that the $\mathrm{I}_{\mathrm{S}}$ will reach a saturated state when drain voltage increases up to when OLED characteristics is more significant.

\section{Conclusion}

In summary, we have reported the characteristics of OLED with an FET structure. The typical FET behaviors in an OLED were obtained from these devices. The unique electric characteristics both OFET and OLED were analyzed. The five layers of organic semiconductors are believed to play different role in OFET and OLED, respectively. Furthermore, its mechanism is presented based on an equivalent 
circuit. The works to investigate the change in recombination zone of device and improve device performance are in process.

\section{Acknowledgements}

This work was supported by the Cooperative Link for Unique Science and Technology for Economy Revitalization (CLUSTER) of the Ministry of Education, Culture, Sports, Science and Technology, Japan, and shanghai excellent teacher program. One author would like to thank the funding support from Shanghai Rising-Star Program (07QA14023). 


\section{References}

1. V. G. Kozlov, V. Bulovic, P. E. Burrows, and S. R. Forrest, Nature (London) 389 (1997) 362.

2. M. Berggren, A. Dodabalapur, and R. E. Slusher, Appl. Phys. Lett. 71 (1997) 2230.

3. B. Wei, N. Kobayashi, M. Ichikawa, T. Koyama, Y. Taniguchi and T. Fukuda, Opt. Exp. $14(2006) 9436$.

4. V. C. Sundar, J. Zaumseil, V. Podzorov, E. Menard, R. L. Willett, T. Someya, M. E. Gershenson and J. A. Rogers, Science 303 (2004) 1644.

5. V. Podzorov, E. Menard, A. Borissov, V. Kiryukhin, J. A. Rogers and M. E. Gershenson: Phys. Rev. Lett. 93 (2004) 086602-1.

6. J. Wang, H. B. Wang, X. J. Yan, H. C. Huang, D. Jin, J. W. Shi, Y. H. Tang, and D. H. Yan. Adv. Fuct. Mater. 16 (2006) 824

7. J. Wang, H. B. Wang, X. J. Yan, H. C. Huang,, and D. H. Yan. Chem. Phys. Lett. 407 (2005) 87.

8. M. Ichikawa, H. Yanagi, Y. Shimizu, S. Hotta, N. Suganuma, T. Koyama and Y. Taniguchi, Adv. Mater. 14 (2002) 1272.

9. A. Hepp, H. Heil, W. Weise, M. Ahles, R. Schmechel and H. von Seggern, Phys. Rev. Lett. 91 (2003) 157406.

10. M. Ahles, A. Hepp, R. Schmechel and H. von Seggern, Appl. Phys. Lett. 84 (2004) 428.

11. C. Rost, S. Karg, W. Riess, M. A. Loi, M. Murgia and M. Muccini, Appl. Phys. Lett. 85 (2004) 1613.

12. T. Oyamada, H. Sasabe, C. Adachi, S. Okuyama, N. Shimoji and K. Matsushige, Appl. Phys. Lett. 86 (2005) 86.

13. K. Nakamura, M. Ichikawa, R. Fushiki, T. Kamikawa, M. Inoue, T. Koyama, and Y. Taniguchi, Jpn. J. Appl. Phys. 44 (2005) L1367. 
14. C. C. Chu, C. W. Chen, S. H. Li, E. H. E. Wu, and Y. Yang. Appl. Phys. Lett. 86 (2005) 253503.

15. K. Okumoto and Y. Shirota, J. Lumin. 87 (2000) 1171.

16. S. Tabatake, S. Naka, H. Okada, H. Onnagawa, M. Uchida, T. Nakano and K. Furukawa, Jpn. J. Appl. Phys. 41 (2002) 6582.

17. B. Wei, K. Furukawa, J. Amagai, M. Ichikawa, T. Koyama and Y. Taniguchi, Semicond. Sci. Tech. 19 (2004) L56. 


\section{Figure captions}

FIG. 1. Configuration of OLEDs with a FET structure of top-contact. Five organic layers acting as different function are showed in OFET and OLED

FIG. 2. The electric and luminescent characteristics of OLED in current studies.

FIG. 3. The typical transistor characteristics of OLEDs with a FET structure. (a) Family curves (b) Transfer characteristics.

FIG. 4. (a) Equivalent circuit of an OLED with a top-contact FET configuration and (b) Electric modulation characteristics. 
Fig.1 Bin Wei el al

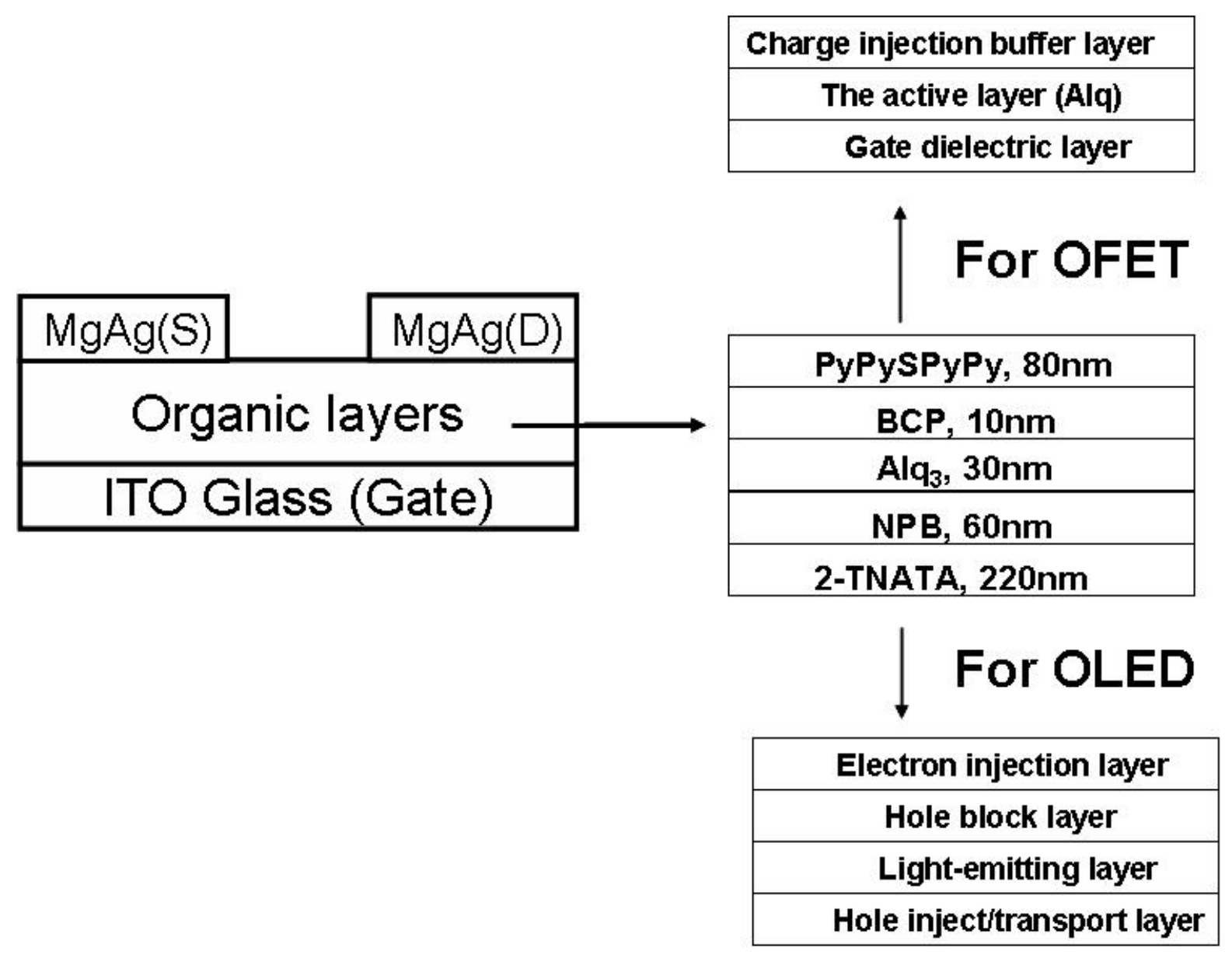


Fig.2 Bin Wei el al

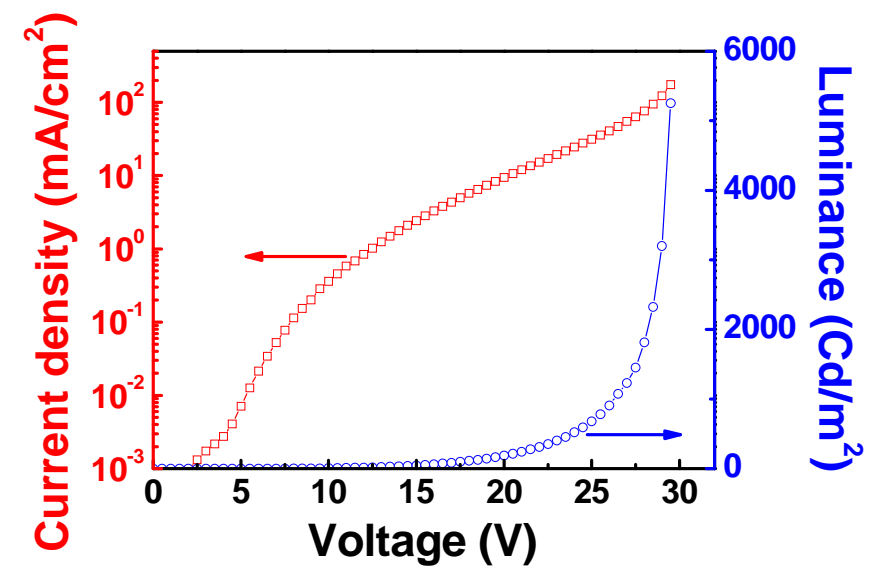


Fig.3 Bin Wei el

a

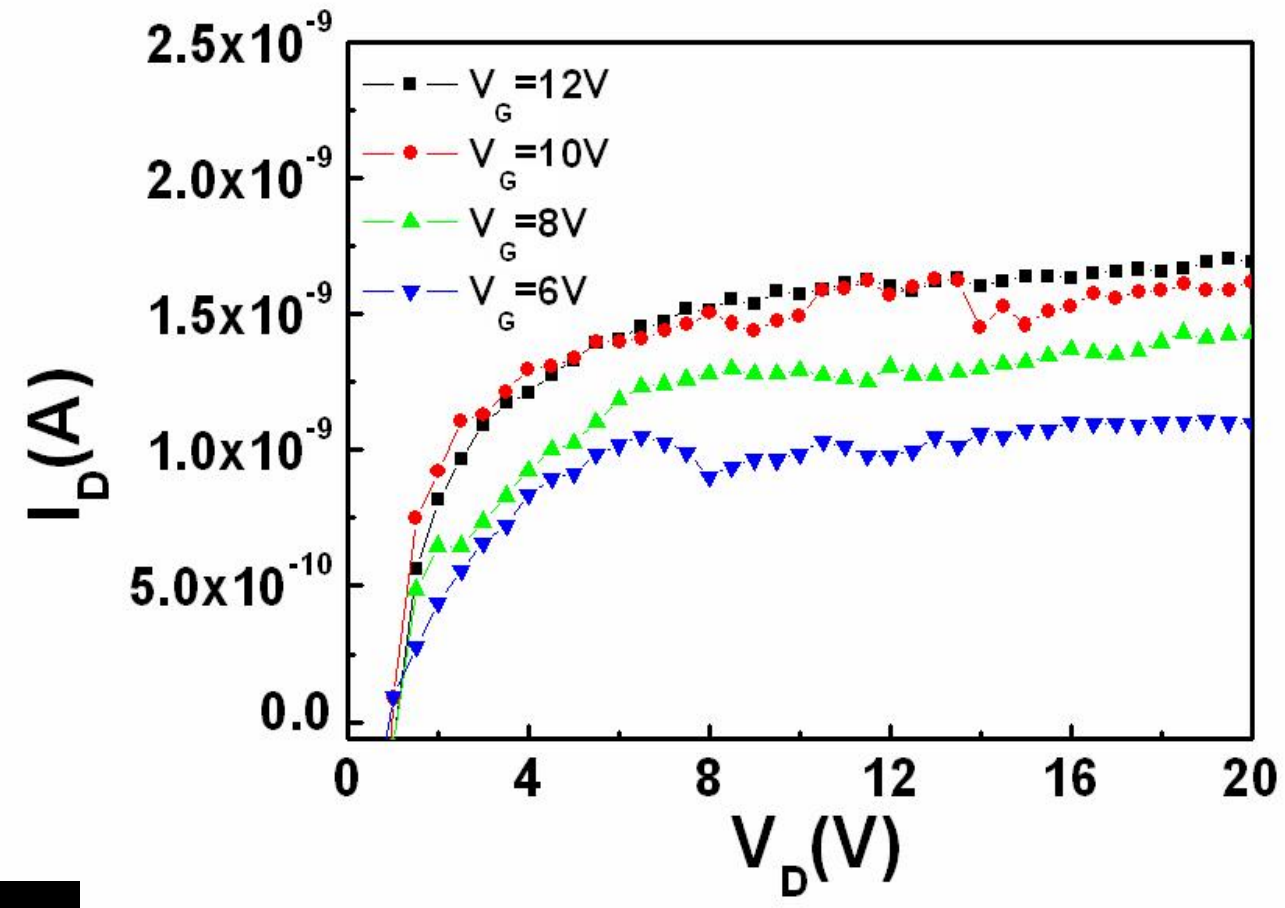

b

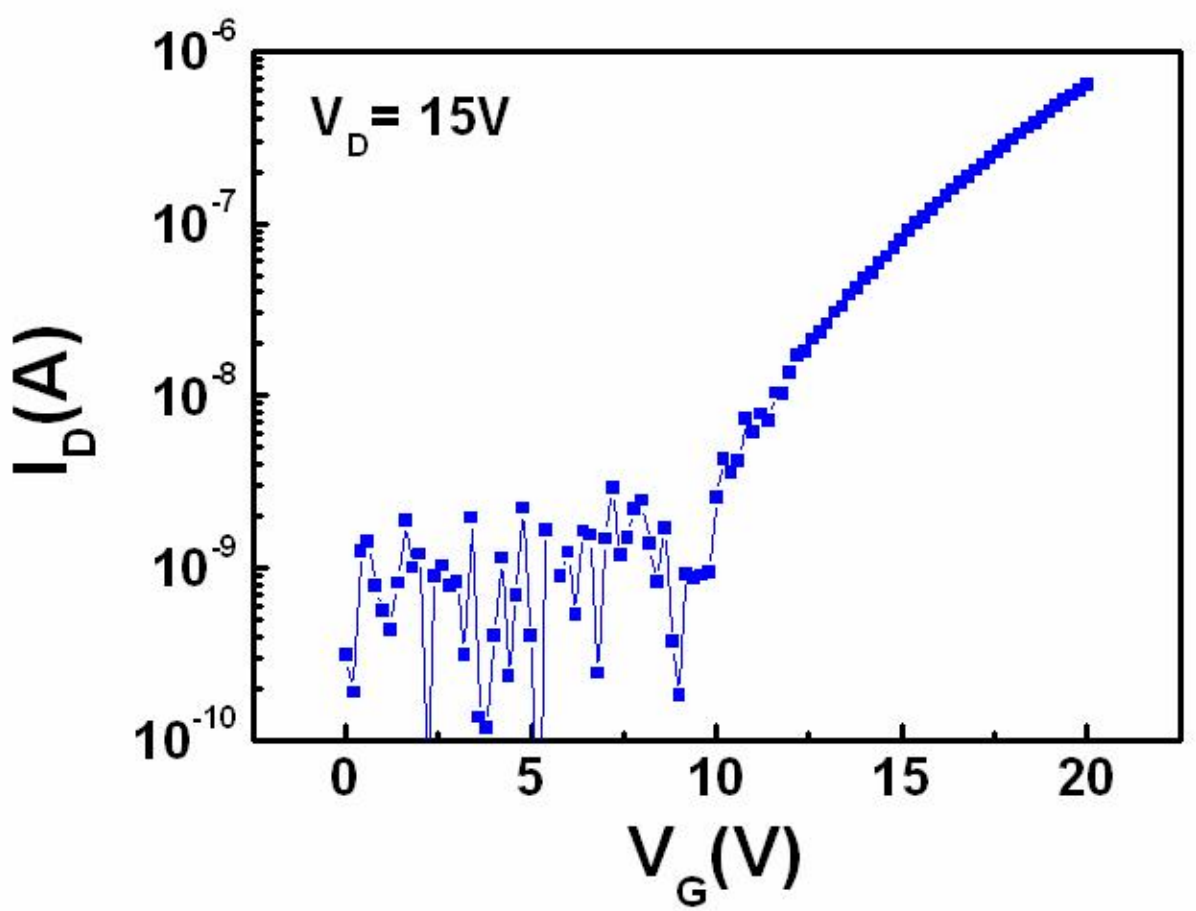


Fig.4 Bin Wei el al
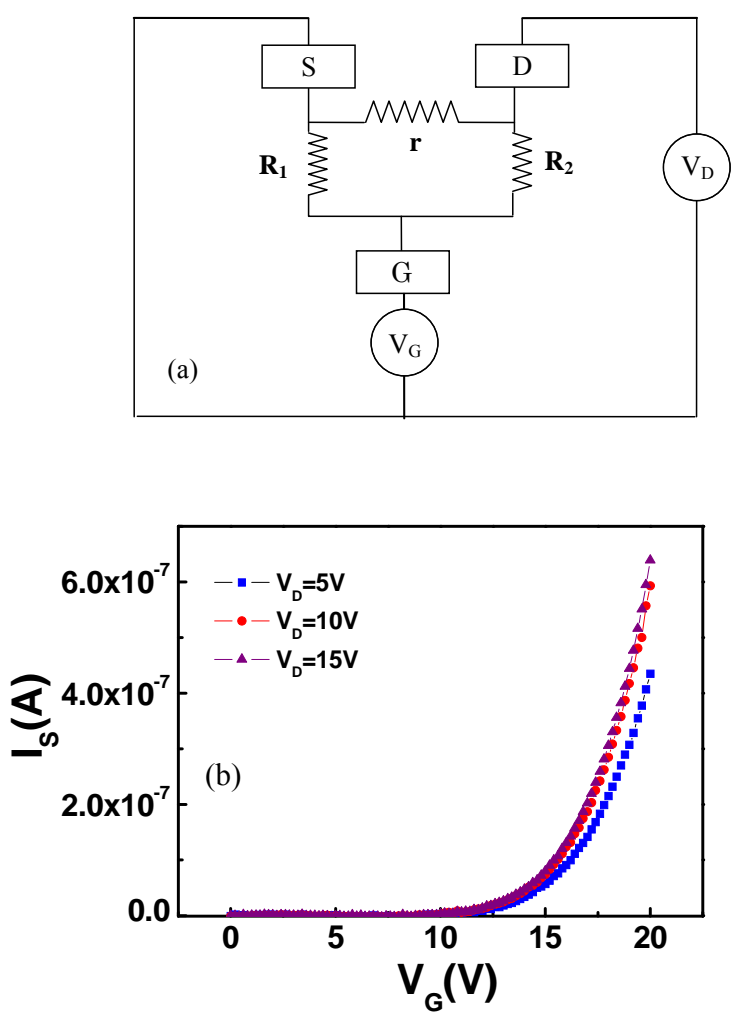